Relations industrielles

Industrial Relations

\title{
Evaluating the Labor-Market Effects of Social Programs, edited by 0 . Ashenfelter et J. Blum, Industrial Relations \\ Section, Princeton University, 176, 238 pp.
}

\section{Jacques Saint-Laurent}

Volume 32, numéro 3, 1977

URI : https://id.erudit.org/iderudit/028815ar

DOI : https://doi.org/10.7202/028815ar

Aller au sommaire du numéro

Éditeur(s)

Département des relations industrielles de l'Université Laval

ISSN

0034-379X (imprimé)

1703-8138 (numérique)

Découvrir la revue

Citer ce compte rendu

Saint-Laurent, J. (1977). Compte rendu de [Evaluating the Labor-Market Effects of Social Programs, edited by O. Ashenfelter et J. Blum, Industrial Relations Section, Princeton University, 176, 238 pp.] Relations industrielles / Industrial Relations, 32(3), 471-472. https://doi.org/10.7202/028815ar

Tous droits réservés @ Département des relations industrielles de l'Université Laval, 1977
Ce document est protégé par la loi sur le droit d'auteur. L'utilisation des services d’Érudit (y compris la reproduction) est assujettie à sa politique d'utilisation que vous pouvez consulter en ligne.

https://apropos.erudit.org/fr/usagers/politique-dutilisation/ 
ture. Cette partie traite également de la formation des dirigeants et du développement organisationnel.

Une revue rapide des études canadiennes nous amène rapidement à constater qu'il existe effectivement de légères différences au niveau des conclusions de la recherche, surtout dans les organisations où l'on retrouve des canadiens d'origine française à différents paliers de la structure. C'est donc que les sciences du comportement sont en quelque sorte liées à une culture donnée et leur degré de généralisation demeure limité. C'est à mon avis, le point le plus intéressant que les textes font ressortir et j'imagine que c'est là la raison d'être de la publication de cet ouvrage.

Université Laval

Laurent BÉLANGER

Guide to Statutory Provisions in Public Sector Bargaining - Impasse Resolutions Procedures, (Second issue) by Helen S. Tanimoto, Hawai, Industrial Relations Center, 1977, $170 \mathrm{pp}$.

Dans le cadre d'une série de Guides des dépositions statutaires traitant de la négociation collective dans le secteur public, le Centre des relations industrielles de l'Université d'Hawaï vient de rééditer avec mise à jour celui qui porte sur les procédures à suivre dans le cas d'impasses.

Les autres Guides publiés antérieurement étaient consacrés à a) l'employeur public et le devoir de négocier; b) la détermination des unités de négociation; c) le droit de grève et ses prohibitions; d) l'organisation des travailleurs et les droits de représentation; e) le statuts des employés de direction, des cadres et de ceux qui détiennent des postes à caractère confidentiel; f) les termes et les définitions.

Dans une première partie, l'auteur fait une analyse et une synthèse de diverses dispositions dans la législation de tous les États des États-Unis: délais d'intervention pour la médiation, l'enquête factuelle et l'arbitrage, qualifications exigées des intervenants, les agences administratives, les procédures facultatives, les critères, les appels, le coût de ces recours.
Dans une deuxième partie, le texte même de la législation de chacun des États est présenté selon le même plan: la détermination de l'impasse, les étapes prévues tant pour la médiation, l'enquête factuelle, l'arbitrage que pour une autre procédure, de même que le genre de rapports ou recommandations qui doit être fait et leur effet obligatoire. Enfin, qui doit défrayer les coûts relatifs à l'utilisation de ces procédures. L'auteur prend la peine d'avertir le lecteur que tous ces textes doivent être envisagés dans le contexte de la loi complète.

Ce Guide, comme les autres, est un instrument extrêmement précieux pour quiconque veut connaître rapidement les dispositions législatives actuellement en vigueur aux États-Unis.

Université Laval

Gérard DION

Evaluating the Labor-Market Effects of Social Programs, edited by O. Ashenfelter et J. Blum, Industrial Relations Section, Princeton University, 1976 238 pp.

Ce volume contient les cinq conférences prononcées à un congrès tenu à l'Université de Princeton au printemps 1974, congrès portant sur les effets des programmes gouvernementaux de bien-être sur le marché du travail.

Le congrès avait pour objectifs:

a) souligner l'importance de la recherche théorique et empirique sur les effets des divers programmes du gouvernement dans le domaine des salaires, de l'emploi et des conditions de travail;

b) et soumettre à la critique les communications présentées afin d'évaluer la solidité de leur logique et de la méthode empirique utilisée. On ne s'étonnera donc pas si toutes ces communications sont hautement théoriques et de lecture peu facile pour ceux qui n'ont pas déjà une bonne formation en théorie et en mathématiques économiques.

Les communications portent sur les sujets suivants: la législation américaine en matière de salaire minimum; les effets de la 
loi contre la discrimination envers les minorités; les effets des programmes de dépenses publiques sur l'emploi; les effets sur la production, l'emploi et les prix du transport, de la responsabilité des paiements aux victimes de la "pneumoconiosis du gouvernement à l'entreprise privée dans l'industrie du charbon; la conceptualisation des avantages possibles de politiques gouvernementales élaborées dans le but de faciliter le placement des travailleurs sans emploi dans les emplois vacants.

Ces communications sont précédées d'une excellente introduction sur les nécessités d'évaluer de tels programmes; sur les difficultés d'établir une base conceptuelle solide pour une telle évaluation; sur les caractéristiques essentielles d'une évaluation valable; sur le processus même d'évaluation; et sur les avantages de telles évaluations.

Jacques SAINT-LAURENT

Université Laval

The Current Industrial Relations Scene in Canada 1977, by H. D. Wood and Pradeep Kumar (ed.). Kingston, Industrial Relations Centre, Queen's University, 1977, (pas de pagination).

À chaque année, le Centre des relations industrielles de l'université Queen's publie sous ce titre un ouvrage compréhensif réunissant la plupart des informations que doivent connaître ceux qui œuvrent dans le domaine des relations du travail au Canada.
L'ouvrage est divisé en sept sections: l'économie; le marché de la main-d'œuvre; la législation du travail et la politique sociale; le syndicalisme; la négociation collective; les salaires, la productivité et les coûts de la main-d'œuvre; la documentation. Chaque section comprend trois parties: un résumé des développements qui se sont produits au cours de l'année précédente; des notes techniques sur les sources d'information, la méthodologie et les définitions utilisées; des tableaux statistiques.

En ce qui regarde l'information factuelle, les auteurs ont rassemblé tout ce qui pouvait être pertinent, mais qui nécessiterait un temps énorme pour consulter une multitude de publications officielles éparses. Quant aux notes techniques, elles possèdent une valeur permanente pour analyser et comprendre les données fournies. Enfin, dans la documentation on a présenté une liste de toutes les publications ouvrages et articles récemment publiés touchant les relations du travail.

Le Centre des relations industrielle de l'université Queen's, tirant profit d'une équipe de chercheurs très compétents et d'une documentation abondante, met à la disposition du public un ouvrage unique dans son genre au Canada que nous considérons comme un instrument absolument indispensable pour tous ceux qui veulent connaitre la situation actuelle des relations du travail. C'est un livre de chevet pour les professeurs, les élèves et les praticiens.

Université Laval
Gérard DION 